\title{
The Determinants of Optional Insurance Coverage with Flexible Spending Accounts in Post-Affordable Care Act-USA
}

\author{
Ibrahim Niankara \\ College of Business, Al Ain University of Science and Technology, P.O. Box: 112612, Abu Dhabi, UAE; Tel: \\ +97124444696 Ext: 539 ; Fax: +97124444304 ; E-mail: ibrahim.niankara@aau.ac.ae
}

\begin{abstract}
Motivated by the theoretical model of health insurance choice with Flexible Spending Accounts (FSAs) presented in Cardon (2012), this study investigates the determinants of optional coverage (SSP) and flexible spending accounts (FSA) enrollment, among the privately insured in post-affordable-care-act (ACA) USA. To this end, we rely on semi-parametric bi-variate probit methods, along with a pooled crosssection of the 2015-2016 National Health Interview Surveys. As predicted by the theoretical model, we find that SSP and FSA are complement health solutions with a positive correlation. Our results emphasize that the most important trigger factors influencing the joint probability of SSP and FSA adoption include not only insurance premium cost, but also age, education, marital status, number of work hours, region of residency, citizenship status, and annual health expenditure level. We find that controlling for these latter factors, health status is not significant especially for FSA adoption. In addition, despite the fact that the relative frequency of individuals with FSA rises with increasing levels of medical expenditure, ACA restrictions on FSA tax exclusion to an annual adjusted maximum of $\$ 2600$ (in $2017 \$$ s) seems to adversely burden individuals with greater medical expenditure, thereby reducing their likelihood of FSA enrollment in post-ACA USA. Understanding these factors is very crucial to US health care market's stakeholders, including insurance companies, firms looking to design their health insurance offerings, but also policy-makers interested in providing new tailored health solutions for reducing health risks.
\end{abstract}

Keywords: Affordable Care Act, Flexible Spending Accounts, Insurance Coverage, Multiple Service Plans

JEL: I13, I18, C14, C25, C35, D01 


\section{Introduction}

Optional coverage, according to the US Medical Expenditure Panel Survey (MEPS) insurance component glossary of health insurance terms, represents any separate coverage that insured individuals adopt for a limited area of medical care to supplement their basic health insurance plan. Also referred to as "Single Service Plans - (SSPs)" they require an additional premium to be paid by the enrollee and/or the employer for the additional coverage. Flexible Spending Accounts (FSAs) on the other hand are a widely used arrangement, introduced under a 1978 policy that allow employees to set aside pre-tax income to pay for qualified out-of-pocket health expenses not covered by employer-provided health insurance, hence making health care cheaper ex post (Cardon and Showalter, 2001). Employer-provided health insurance was first subsidized in 1954 when premiums paid by employers on behalf of their employees were exempted from tax, inducing individuals to buy more generous insurance than they otherwise would (Pauly, 1986). In 1978, Section 125 of the Internal Revenue Code extended the tax exemption for health insurance to fully subsidize not only premium costs, but also out-of-pocket payments through the use of FSAs. Individuals with access to FSAs can make periodic tax-free contributions, which can then be used to reimburse qualified costs, including deductibles, co-payments, and coinsurance obligations that are part of an insurance policy, or simply to pay for uninsured medical expenses (Jack et al., 2006). The initial structure of FSAs included a significant forfeiture risk if households had unused funds in their accounts at the end of the year (Ramsay and Oguledo, 2011). This later characteristic has been subject to many controversy, and in 2015 the US treasury made an administrative ruling offering a grace period for spending the funds after the end of the calendar year. This ruling reduced significantly the forfeiture risk to FSA participants, and along federal government tax revenues (Cardon et al. 2013). The inaction of the Patient Protection and Affordable Care Act (ACA) 1 , and the Education Reconciliation Act have brought changes that limit the tax exclusion from FSA to an inflation adjusted value of $\$ 2600$ in 2017 dollars (Cardon et al., 2012). Although this limit was estimated to increase federal payroll and income tax receipts by $\$ 13$ billion between 2013 and 2019, the welfare implications of this change in terms of its effects on individuals' enrollment incentives are still relatively undocumented.

Indeed, the literature on consumer and firm behavior with respect to FSAs are fairly sparse Cardon and Showalter, 2001, 2003, Hamilton and Marton, 2008; Ramsay and Oguledo, 2011;

\footnotetext{
${ }^{1} \mathrm{ACA}$ is the healthcare law passed by Congress on March 3, 2010 during the administration of President Barack Obama, with the aim of ensuring that most Americans can receive or buy health insurance (Harrington, 2010). Phased in over four years from 2011 to 2015, ACA constituted what is widely considered the most sweeping change to the U.S. healthcare system since Medicare and Medicaid were created in 1965 (Even and Macpherson, 2016). Together the Individual mandate and Company mandate of the Act changed not only healthcare sector dynamics, but also labor market dynamics in the US Niankara $(2018 d)$.
} 
Cardon, 2012; Cardon et al., 2012, 2013), and provides relatively more theoretical treatment of FSAs adoption in a variety of context, compared to the empirical literature (Cardon and Showalter, 2001; Feldman and Schultz, 2001; Jack et al., 2006; Hamilton and Marton, 2008), which lags behind, due to the difficulty associated with obtaining consumer-level data on FSA use. Therefore, this paper extends the empirical literature on FSA, using a pooled cross-section of the 2015-2016 National Health Interview Surveys (NHIS), to provide a demand side counterpart to the analysis in (Niankara, 2018c) by investigating the determinants of optional coverage (SSP) and FSA choices in post-ACA-USA. Since FSAs cover out-of-pocket costs, their availability should have predictable effects on individuals' coverage choices. Hence understanding what factors influence this joint choice is very crucial to all stakeholders, including companies looking into designing their health insurance offerings, insurance companies, health practitioners, but also policy-makers interested in providing new tailored health solutions for reducing health risks.

Furthermore, in his theoretical treatment of health insurance choice with flexible spending accounts, Cardon (2012) shows that FSA participation decisions are made concurrently with insurance plan choices annually during companies' open enrollment period. Therefore these decisions are made jointly, and with the same information. Hence we use this framework as our motivation to investigate the impact of important socio-economic factors on the joint probability of FSAs and SSPs adoption among privately insured Americans in the period post ACA inaction.

As predicted by the theoretical model, we find that the choice of optional coverage positively correlates with increased FSA participation. Our results emphasize that the most important factors influencing the joint probability of optional coverage, and Flexible Spending Account Adoption include not only individual's health insurance premium cost, but also age, education, hours spent working, earnings, marital status, region of residency, citizenship status, and family health expenditure level.

The rest of the paper is organized as follows: In section 2 we present the theoretical model of health insurance choice with flexible spending accounts; section 3 describes the empirical model with four different specifications; section 4 presents the data and the variables used in the econometric modeling; section 5 presents and discusses the results, while section 6 concludes the analysis.

\section{The Theoretical Model of Health Insurance Choice with FSA}

In this section, we briefly describe the theoretical model of health insurance choice with FSA presented in Cardon (2012), which provides motivation for the empirical model presented in the next section. An individual with positive pre-tax income $I$ and marginal tax rate $t$ is assumed to face a loss $x \in[0, L]$, with the maximum possible loss less than $I(1-t)$. Define $g(x)$ as the continuous loss function, with corresponding distribution function $G(x)$, which is assumed to be public information. Assume further that the individual has smooth utility function $U()$ representing 
preferences over consumption $C$, with $U^{\prime}>0$ and $U^{\prime \prime}<0$. The individual is eligible to adopt a flexible spending account (FSA) allowing the coverage of the loss or a portion of it to be paid using FSA funds. The periodic funds $F$ allocated to the FSA account must be committed before the realization of the loss. The portion of the loss in shortage of the committed amount is forfeited, while its portion in excess is not covered. The model assumes a two stage decision process. The first stage corresponds to the choices of coverage type (insurance contract) and an FSA election amount, while the second stage corresponds to the choice of funds withdrawal $W$, subject to realized health expenditure and first stage choices. It's assumed that the coverage types are deductible insurance ${ }^{2}$, with premium $P(D)=(1+\lambda) \int_{D}^{L}(x-D) g(x) d x$, where $\lambda$ is the proportional loading factor, and such that the FSA funds are first used to cover the costs of deductibles.

If the individual is offered a FSA along with deductible insurance coverage, then using integration by parts we have that $P^{\prime}(D)=-(1+\lambda)(1-G(D))$, and the individual's problem becomes that of choosing a level of deductible coverage $D$ along with the amount $F$ allocated to the FSA, prior to observing the loss $x$. Once the loss is realized, the individual chooses a withdrawal, $W$, which must be less than or equal to $F$ and to the total out-of-pocket medical expenses. Therefore, conditional on $D, F$ and $x$, the individual consumption function is $C=(I-P(D)-F)(1-t)+W-\min \{w, D\}$, subject to $W \leq F$ and $W \leq \min \{x, D\}$. The optimization problem is therefore:

$$
\begin{array}{ll}
\max _{D, F, W} & E(U(C) \\
\text { subject to } & C=(I-P(D)-F)(1-t)+W-\min \{w, D\}, \\
& W \leq F, \\
& W \leq \min \{x, D\},
\end{array}
$$

With non-negativity constraints $F \geq 0$ and $D \geq 0$.

$$
W^{*}(x)= \begin{cases}x & \text { if } x<F \leq D \\ F & \text { if } F \leq x<D \\ \min \{F, D\} & \text { if } \mathrm{x} \geq \mathrm{D} \text { and } \mathrm{x} \geq \mathrm{F} .\end{cases}
$$

Since FSA balances $F$ in excess of the deductible $D$ are forfeited, we have that $F \leq D$, and we can define $C_{1}=(I-P-F)(1-t)$ for $x<F, C_{2}=(I-P)(1-t)+1 F-x$ for $F \leq x<D$, and $C_{3}=(I-P)(1-t)+t F-D$ for $x \geq D$, implying $C_{1} \geq C_{2}>C_{3}$. The expected utility is therefore a linear combination

$$
E U=\int_{0}^{F} U\left(C_{1}\right) g(x) d x+\int_{F}^{D} U\left(C_{2}\right) g(x) d x+\int_{D}^{L} U\left(C_{3}\right) g(x) d x
$$

\footnotetext{
${ }^{2}$ A type of insurance contract in which the beneficiary covers the first dollars "deductible" of health care services before the insurance plan starts to pay
} 
with the Lagrangian given as:

$$
\ell(D, F, \mu)=E U+\mu(D-F)
$$

and the Kuhn-Tucker conditions, with complementary slackness (w.c.s.) are given by:

$$
\begin{gathered}
\ell_{D}=\int_{0}^{F} U^{\prime}\left(C_{1}\right)(t-1) P^{\prime}(D) g(x) d x+\int_{F}^{D} U^{\prime}\left(C_{2}\right)(t-1) P^{\prime}(D) g(x) d x \\
+\int_{D}^{L} U^{\prime}\left(C_{3}\right)\left[(t-1) P^{\prime}(D)-1\right] g(x) d x+\mu \leq 0 \text { and } D \geq 0 \text { w.c.s. } \\
\ell_{F}=\int_{0}^{F} U^{\prime}\left(C_{1}\right)(t-1) g(x) d x+\int_{F}^{D} U^{\prime}\left(C_{2}\right) t g(x) d x \\
+\int_{D}^{L} U^{\prime}\left(C_{3}\right) t g(x) d x+\mu \leq 0 \text { and } F \geq 0 \text { w.c.s., } \\
\ell_{\mu}=D-F \geq 0 \text { and } \mu \geq 0 \text { w.c.s., }
\end{gathered}
$$

Solving for the individual's optimal deductible and FSA election amounts, $D^{*}$ and $F^{*}$, gives the following:

(i) $\forall t>0$ and $\lambda>0, D^{*}=F^{*}>0$ and $G\left(D^{*}\right)=\lambda /(1+\lambda) \Longleftrightarrow(1-t)(1+\lambda) \leq 1$.

That is, given a fair or better insurance policy, the individual chooses a full insurance coverage, and also contributes an amount equal to her insurance deductible in FSA.

(ii) $\forall t>0$ and $\lambda>0, D^{*}>F^{*}>0 \Longleftrightarrow(1-t)(1+\lambda)>1$.

That is, given an unfair insurance policy, the individual chooses partial insurance coverage, and contributes an amount less than her insurance deductible in FSA.

(iii) $\forall t=0$ and $\lambda>0, \Rightarrow D^{*}>F^{*}=0$.

That is, when FSA offers forfeiture risk for unused funds, with no reward in return, then the individual gets a partial deductible coverage, but opt out of FSA subscription.

(iv) $\forall \lambda \leq 0, \Rightarrow D^{*}=F^{*}=0$.

That is, if the employer pays any portion of the insurance premium, the individual opt out of both, deductible coverage, and FSA subscription. 
The qualitative features of the first two optimality conditions (i) and (ii) are identical as both lead to the individual opting for both deductible insurance coverage and FSA. The probability of their realization is :

$$
\operatorname{Pr}[\text { Coverage } \cap F S A]=P\left[D^{*}=F^{*}>0 \mid t>0, \lambda>0\right]+P\left[D^{*}>F^{*}>0 \mid t>0, \lambda>0\right]
$$

Our empirical investigation as described next is concerned with identifying the factors determining this joint probability, given a pool of privately insured health consumers from the 2015-2016 National Health Interview Surveys.

\section{The Empirical Model}

Our econometric model of single service plans (SSPs) and flexible spending accounts (FSAs) adoption is grounded on the Random Utility framework following Marshak (1959); Manski and McFadden (1981); Train (2009); Niankara (2018b). It is assumed that each individual is faced with two choice situations $i$ (with $i=1$ if choice of optional coverage, and $i=2$ if choice of flexible spending account, where (s)he must choose between two alternatives indexed respectively with zero (0) and one (1), according to which one provides the greatest utility (or well-being). In the first situation the choice is between 1- "Adopting optional coverage", and 0-" Not-adopting optional coverage". In the second situation, the choice is between 1- "Adopting a flexible spending account", and 0 - "Not-adopting a flexible spending account". In each choice situation the individual chooses the alternative with the highest utility.

Allowing individuals to choose their health insurance coverage and FSA contribution is also theoretically supported by models of benefit provision (Dranove et al., 2000: Gruber and McKnight, 2003), which assume that individuals sort across employment alternatives based on heterogeneous tastes for insurance and FSA contributions. Therefore in the first choice situation, the discrete outcome variable $y_{1}=1$ if adopting optional coverage provides the individual with the greatest utility, otherwise $y_{1}=0$. Similarly in the second choice situation, the discrete outcome variable $y_{2}=1$ if adopting a flexible spending account provides the individual with the greatest utility, otherwise $y_{2}=0$. The utility formulation for alternatives 0 and 1 in each choice situation $i$ for $i=1,2$ can be presented as:

$$
\begin{aligned}
& U_{i 0}=V_{i 0}+\epsilon_{i 0}, \\
& U_{i 1}=V_{i 1}+\epsilon_{i 1},
\end{aligned}
$$

where $V_{i 0}$ and $V_{i 1}$ are deterministic components of utility with $\epsilon_{i 0}$ and $\epsilon_{i 1}$ representing the random components. We observe the event "Adopting optional coverage, and a flexible spending account", $y_{i}=1$ iff $U_{i 1}>U_{i 0}$, that is if and only if alternative 1 in choice situation $i$ has the highest 
utility of the two. Because of the presence of the random components in the utility functions, this event is also random with probability given as:

$$
\begin{aligned}
\operatorname{Pr}\left[y_{i}=1\right] & =\operatorname{Pr}\left[U_{i 1}>U_{i 0}\right] \\
& =\operatorname{Pr}\left[V_{i 1}+\epsilon_{i 1}>V_{i 0}+\epsilon_{i 0}\right] \\
& =\operatorname{Pr}\left[\epsilon_{i 0}-\epsilon_{i 1}<-\left(V_{i 0}-V_{i 1}\right)\right] \\
& =F\left(V_{i 0}-V_{i 1}\right)
\end{aligned}
$$

with $F($.$) representing the cumulative distribution function of the error differences \left(\epsilon_{i 0}-\epsilon_{i 1}\right)$, such that:

$$
\operatorname{Pr}\left[y_{i}=1\right]=F\left(X^{\prime} \beta_{i}\right) \text { if } V_{i 0}-V_{i 1}=X^{\prime} \beta_{i}
$$

Different Discrete Choice Models (DCM) are obtained from different parametric assumptions about the distribution $F($.$) of the error differences \left(\epsilon_{i 0}-\epsilon_{i 1}\right)$. For example the Logit model or logistic regression is obtained when we assume that $F($.$) follows the type 1$ extreme value cumulative distribution, in which case $F\left(X^{\prime} \beta_{i}\right)=\Lambda\left(X^{\prime} \beta_{i}\right)$. On the other hand the probit model or probit regression is obtained when we assume that $F($.$) follows the normal cumulative distribution,$ in which case $F\left(X^{\prime} \beta_{i}\right)=\Phi\left(X^{\prime} \beta_{i}\right)$.

Next we present few specifications of this general behavioral model, starting with the most restrictive representation of the choice probabilities in equation (10), and sequentially relaxing each restriction to end up with the most general model representation for the determinants of optional coverage and flexible spending account adoption in the USA.

\subsection{Univariate Unadjusted Probit Specifications}

This first specification is the most restrictive of our series of model representations and assumes that $F\left(X^{\prime} \beta_{i}\right)=\Phi\left(X^{\prime} \beta_{i}\right)$, such equation equation 10 can be rewritten for all $i$ independents, with $i=1,2$ as:

$$
\operatorname{Pr}\left[y_{i}=1\right]=\Phi\left(X^{\prime} \beta_{i}\right)=\Phi\left(\beta_{i 0}+\beta_{i 1} \text { PremCost }\right)
$$

Given that the insurance premium equation in the theoretical model is a function of the decision variables, the specification in equation (11) indicates that insurance premium alone is sufficient for predicting the probabilities of optional coverage and flexible spending accounts adoption. The objective is then to estimate the effects of premium costs $\beta_{i 1}$, and the intercept values $\beta_{i 0}$

\subsection{Univariate Adjusted Probit Specifications}

In the second specification, we relax the restriction of insurance premium being the only relevant predictor of the probabilities of optional coverage and flexible spending account adoption, and 
adjust for the effects of other covariates also influencing these probabilities. With this adjustment equation (11) becomes for all independents $i$, with $i=1,2$ :

$$
\operatorname{Pr}\left[y_{i}=1\right]=\Phi\left(X^{\prime} \beta_{i}\right)=\Phi\left(\beta_{i 0}+\beta_{i 1} \text { PremCost }+\beta_{i 3}^{\prime} \text { ContVars }_{i}\right)
$$

where ContVars $s_{i}$ represents the vector of control variables in each choice situation $i$, and capturing individuals' health and socio-demographic characteristics, while $\beta_{i 3}^{\prime}$ is the matrix of their estimated effects.

\subsection{Fully-Parametric Bivariate Probit Specification}

This third specification relaxes the independence assumption made in the previous two, and allows for correlation between the process of optional coverage adoption, and the process of flexible spending account adoption such that $\Phi()$ is the bivariate normal distribution, with the joint probability of optional coverage and flexible spending account adoption given for all dependent $i$ with $i=1,2$ as:

$$
\operatorname{Pr}\left[y_{i}=1\right]=\Phi\left(X^{\prime} \beta_{i}\right)=\Phi\left(\beta_{i 0}+\beta_{i 1} \text { PremCost }+\beta_{i 3}^{\prime} \text { ContVars }_{i}\right)
$$

The joint distribution of the error terms $\epsilon_{1}$ and $\epsilon_{2}$ is given by:

$$
\left(\begin{array}{l}
\epsilon_{1} \\
\epsilon_{2}
\end{array}\right) \sim N\left[\left(\begin{array}{l}
0 \\
0
\end{array}\right),\left(\begin{array}{cc}
1 & \sigma_{12} \\
\sigma_{12} & \sigma_{2}^{2}
\end{array}\right)\right]
$$

Where the variance of the optional coverage adoption process is set to $\sigma_{1}^{2}=1$ for identification purposes following Train $(2009)$. And our objective is same as in the uni-variate adjusted probit case in equation (12), with the distinction that here, in addition to the estimated effects of the variables included in the model, we also need to estimate the values of the correlation coefficient $\sigma_{12}$, and the variance of the flexible spending account adoption process $\sigma_{2}^{2}$. Identification of all model parameters is achieved using the two step estimator by Heckman (1979) described in (Cameron and Trivedi, 2005, 547-548).

\subsection{Semi-Parametric Bivariate Probit Specification}

This fourth and last specification takes the third specification and further relaxes the linearity assumption for all the numerically entering explanatory variables (PremCost, Age, Education, and Hours Worked) in the process of the joint adoption of optional coverage and flexible spending account. We achieve this by specifying general copula functions for these variables $g_{i 1}$ (PremCost), $g_{i 2}($ Age $), g_{i 3}$ (Education) and $g_{i 4}(W R K H R S)$, which are then estimated along with the remaining parameters as described in the fully-parametric case. We adopt this approach because some of the variables embody productivity and life-cycle effects that are likely to have non-linear influences 
on the binary outcomes(Wojtys et al., 2016). Imposing a priori linear relationship as in the fullyparametric specification could mean failing to capture the true and more complex relationships. Also, the bivariate normality implicitly assumed in the fully-parametric specification may be too restrictive for applied work and is typically made for mathematical convenience. Hence the general form of the equation (13) becomes:

$$
\left\{\begin{aligned}
\operatorname{Pr}\left[y_{i}=1\right] & =\Phi\left(X^{\prime} \beta_{i}\right) \\
& =\Phi\left[\beta_{i 0}+g_{i 1}\left(\text { PremCost }^{\prime}+g_{i 2}(\text { Age })+g_{i 3}(\text { Education })+g_{i 4}(\text { WRKHRS })\right.\right. \\
& \left.+\beta_{i 5}^{\prime} \text { NomContVars }_{i}\right]
\end{aligned}\right.
$$

where NomContVars $s_{i}$ represents the vector of nominal control variables in choice situation $i$, while $\beta_{i 5}^{\prime}$ captures their effects on the joint adoption of optional coverage and flexible spending account. The model is called partially linear, and is estimated in R Statistical Software ( $\mathrm{R}$ Core Team, 2015) using the semi-parametric methods presented in Wojtys et al. (2016).

\section{Data}

The data used in this analysis is a pooled cross section of the 2015-2016 National Health Interview surveys (NHIS) (National Center for Health Statistics and others, 2015, 2016). The publicly released data files for the 2015 NHIS data has 41,493 households containing 103,789 persons in 42,288 families, while the 2016 sample data concerns 40,220 households containing 97,169 persons in 40,875 families. The conditional response rate for the 2015 Sample Adult component was $79.7 \%$, which was calculated by dividing the number of completed Sample Adult interviews $(33,672)$ by the total number of eligible sample adults $(42,270)$. The unconditional or final response rate of $55.2 \%$ for the Sample Adult component was calculated by multiplying the conditional rate of $79.7 \%$ by the final family response rate $69.3 \%$ (National Center for Health Statistics and others, 2015). Similarly, the conditional response rate for the Sample 2016 Adult component was 80.9\%, which was calculated by dividing the number of completed Sample Adult interviews $(33,028)$ by the total number of eligible sample adults $(40,848)$. The unconditional or final response rate of $54.3 \%$ for the Sample Adult component was calculated by multiplying the conditional rate of $80.9 \%$ by the final family response rate 67.1\% (National Center for Health Statistics and others, 2016). After variables selection, data treatment, and accounting for missing information, the pooled crosssectional panel data used in our final analysis contains a total of 32397 observations, 16028 of which from the 2015 sample adult component and 16369 from the 2016 sample adult component.

In order to achieve our study goal of investigating the determinants of the decision to enroll in complementary coverage to basic health insurance in the presence of flexible spending accounts, our study sample is a random draw from the restricted population of all privately insured americans 
during the fiscal years 2015 and 2016, as provided by the NHIS. For a detailed description of this data-set, consult(Niankara, 2018d). Table (1)provides definitions and summary statistics for the variables used in the analysis.

\section{Results and Discussions}

The results which describe the characteristics of the random sample from the restricted population of individuals with private coverage in the USA in 2015 and 2016, are divided into two major parts. The first part presents the uni-variate descriptive statistics for the variables used in the analysis, followed by the bi-variate descriptive statistics with chi-square tests of independence(for the nominal explanatory variables), and the welch two-sample t-test of difference in groups means (for the numerical explanatory variables). The second part presents and discusses the results of the various econometric specifications.

\subsection{Uni-variate Descriptive Statistics}

The uni-variate descriptive results presented in table (1) show that the average respondent in the pooled sample is middle aged (44.92 years), with at least a bachelor degree (16.15 level of education), and works full-time (41.4 hours) on a weekly basis, while paying annually an average of $\$ 3951$ for premium costs. This mean annual premium cost is $\$ 3876$ in 2015 but increases to $\$ 4024$ in 2016. Table (1) also shows that $49.5 \%$ of the respondents in the pooled sample come from the 2015 NHIS sub-sample, while the remaining 50.5\% come from the 2016 NHIS sub-sample. Furthermore $50.8 \%$ of the respondents in the pooled sample are males, while the remaining $49.2 \%$ are females. With regards to ethnicity, we note that the majority (82.8\%) are Caucasians (whites), followed by $9.6 \%$ blacks, $6.5 \%$ Asians, and finally $1.2 \%$ for other races. With regards to marital status, we note that $65.7 \%$ of the respondents are currently married, $11.9 \%$ were previously married, while $22.4 \%$ never got married. The regional distribution suggests that $33.4 \%$ of the respondents come from the southern part of the United States, $17 \%$ from the northwest, $23.4 \%$ from the Midwest, and the remaining $26.2 \%$ from the west. The place of birth result shows that $84.7 \%$ of the respondents are born inside of the USA, against $15.2 \%$ born outside the country. In terms of citizenship, $94.6 \%$ of the respondents are US citizens, while $5.4 \%$ of them are non-US citizens.

In relation to optional coverage choice, the pooled data results in table (1) show that $56.5 \%$ of the respondents have optional coverage, while the remaining $43.5 \%$ reports having only basic insurance coverage. The proportion of individuals with optional coverage has increased from $54.5 \%$ in 2015 to $58.5 \%$ in 2016. Similarly the proportion of individuals with FSA has increased from $23.4 \%$ in 2015 to $24.3 \%$ in 2016 , with a pooled sample average of $23.8 \%$. In terms of medical costs, table (1) shows that $33.1 \%$ of the respondents in the pooled sample have annual family expenditure less than $\$ 500,35.1 \%$ have family expenditure between $\$ 500$ and $\$ 2000$, while the remaining $31.8 \%$ have family expenditure above $\$ 2000$. The figures on annual earnings also shown in table (1) 
suggest that $33.8 \%$ of the respondents in the pooled sample have earnings below $\$ 35000,35.2 \%$ have earnings between $\$ 35000$ and $\$ 65000$, while the remaining $30.7 \%$ have earnings above $\$ 65000$. The distribution of individuals by physical health status suggests that $33.2 \%$ of respondents report an excellent physical health status (PHS), 38.5\% reports a very good PHS, $23.6 \%$ reports a good PHS, while the remaining $4.7 \%$ reports having fair or poor PHS. Finally $96 \%$ of the respondents reports not being limited in anyway physical, mental or emotional, against only $4 \%$ that report having such limitations.

\subsection{Bi-variate Descriptive Statistics}

In the bivariate case, we begin with the description of the link between the two dependent variables (SSP, and FSA) with each of the nominal explanatory variables in the model using conditional frequency distributions with chi-squared tests of independence; then we turn to their link with the numerical explanatory variables using welch's two-sample t-test of difference in group means.

\subsubsection{The associations with the nominal predictors}

As shown in table (2), the chi-square test results suggest that all the nominal explanatory variables are significantly linked to the dependent variables SSP and FSA since the corresponding p-values are all less than the $5 \%$ level. The only exception comes from the variable capturing respondents' physical mental or emotional limitations which seem to be independent of FSA adoption.

The conditional frequency distributions in table (2) show that the relative frequency of respondents with optional coverage (SSP) is $30.6 \%$ for respondents with annual medical expenditure below $\$ 500$, and rises to $35.8 \%$ for those with annual expenditure between $\$ 500$ and $\$ 2000$, and decreases to $33.5 \%$ for respondents with annual family expenditure above $\$ 2000$. A similar pattern is observed for increasing levels of annual earnings. Indeed the frequency of individuals with optional coverage is $29.3 \%$ among respondents with annual earnings lower than $\$ 35,000$, and rises to $35.8 \%$ for those with annual earnings between $\$ 35,000$ and $\$ 65,000$, and finally falls to $34.9 \%$ for those with annual earnings above $\$ 65,000$. Table (2) also shows that the frequency of respondents with optional coverage increased from $47.7 \%$ in 2015 to $52.3 \%$ in 2016 . However, the frequency of males with optional coverage $(49.4 \%)$ in the pooled sample is less than that of females $(50.6 \%)$. Similarly the greatest share of respondents with optional coverage are Caucasians at 84\%, followed by blacks at $8.7 \%$, then by Asians at $6.4 \%$, and finally other races at $1 \%$. With regards to marital status, table (2) shows that the greatest share of respondents with optional coverage are currently married (67.5\%), followed by those that never married $(21.2 \%)$ and finally by those that were previously married $(11.4 \%)$. The regional distribution of the respondents with optional coverage in the pooled sample suggests that the greatest majority live in the southern states 
(31.5\%), followed by the western states (25.7\%), then by the Midwestern states (24.5\%), and finally by the Northwestern states (18.3\%). Looking at the place of birth, we note that the majority of respondents with optional coverage are born in the united states (87.3\%) against $12.7 \%$ born outside of the country. Similarly the majority of the respondents with optional coverage in the pooled sample are identified as US citizens (95.9\%), against only $4.1 \%$ that are non-US citizens. With respect to physical health status, the greatest share of individuals with optional coverage report a very good $(39.3 \%)$ health status, followed by those reporting an excellent health status at $32.9 \%$, then by those reporting a good health status at $23.4 \%$, and then by those reporting a fair or poor health status at $4.4 \%$. Finally among those with optional coverage in the pooled sample, the majority (95.8\%) report having no physical, mental or emotional limitations, against $4.2 \%$ that report having such limitations.

Now turning to the conditional frequency results for the respondents with FSA as also shown in table (2) we note that the relative frequency of respondents with FSA increases with rising levels of annual medical expenditure. In fact the smallest frequency $(23.8 \%)$ is recorded for respondents with annual medical expenditure below $\$ 500$, followed by those with annual expenditure between $\$ 500$ and $\$ 2000$ at $35.7 \%$, and by those with annual family expenditure above $\$ 2000$ at $41.4 \%$. A similar increasing pattern is observed for increasing levels of annual earnings. Indeed the frequency of individuals with FSA is the smallest (23.8\%) among respondents with annual earnings lower than $\$ 35,000$, followed by those with annual earnings between $\$ 35,000$ and $\$ 65,000$ at $(35.5 \%)$, and finally by those with annual earnings above $\$ 65,000$ at a frequency of $40.8 \%$. Comparing the two years, we note that the frequency of respondents with FSA increased from $48.5 \%$ in 2015 to $51.5 \%$ in 2016 . However, the frequency of males with FSA $(49.8 \%)$ in the pooled sample is smaller than that of females $(50.2 \%)$. Similarly the greatest share of respondents with FSA in the pooled sample are Caucasians at $84.1 \%$, followed by blacks at $8.7 \%$, then by Asians at $6.4 \%$, and finally by other races at $0.8 \%$. With regards to marital status, table (2) shows that the greatest share of the respondents with FSA are currently married (70.3\%), followed by those that never got married $(20.1 \%)$ and finally by those that were previously married (9.6\%). The regional distribution of the respondents with FSA in the pooled sample suggests that the greatest majority live in the southern states $(30.5 \%)$, followed by the Midwestern states $(26.1 \%)$, then by the western states $(25.7 \%)$, and finally by the northwestern states $(17.7 \%)$. Looking at the place of birth, we note that the majority of the respondents with FSA are born in the united states (88.7\%) against 11.3 $\%$ born outside of the country. Similarly the majority are US citizens (96.8\%), against only $3.2 \%$ that are non-US citizens. With respect to physical health status, the greatest share of individuals with FSA report a very good $(39.9 \%)$ health status, followed by those reporting an excellent health status at $34.1 \%$, then by those reporting a good health status at $22.3 \%$, and then those reporting a fair or poor health status at $3.7 \%$. Finally among those with FSA in the pooled sample, the majority $(96.2 \%)$ report having no physical, mental or emotional limitations, against $3.8 \%$ that report having such limitations. 


\subsubsection{The associations with the quantitative predictors}

In order to describe the relationship between the quantitative predictors and the two binary dependent variables SSP and FSA, we first implement Levine's test of homogeneity in variances as shown in table (3), followed by Welch two-sample t-test of difference in means as shown in table (4). The F-statistics with corresponding p-values of the Levine's test provide enough evidence to reject the null of equal variances, and conclude that the variance between individuals with only basic coverage differs significantly from that of individuals with optional coverage. Similarly the variance between individuals with FSA differs significantly from that of those without FSA. These results further suggest that we can proceed to implement our two samples t-test of difference in group means assuming unequal variances. The results of such tests are Indeed presented in table (4) for all the included quantitative variables in the models.

Starting with respondents' coverage status, the results suggest that the average annual premium cost for those with optional coverage is relatively lower at $\$ 3893$ than that of those with only basic coverage at $\$ 4025$. These groups' mean premium expenses are significantly different as shown by the $95 \%$ confidence interval $(-\$ 211,-\$ 53)$ which is void of zero. However the average weekly hours spent working for respondents with optional coverage (41.85 hours) is significantly higher than that of individuals with only basic coverage ( 40.83 hours) as shown by the $95 \%$ confidence interval $(0.75,1.29)$ on the mean difference, which does not contain zero. Furthermore, the mean age of respondents with optional coverage (44.09 years) is also significantly lower than that of respondents with only basic coverage (44.85 years) as indicated by the $95 \%$ confidence interval (-1.05 years, -0.48 years) on the difference in means which is void of zero. Finally the mean level of education for respondents with optional coverage (16.41) is significantly higher than that of respondents with only basic coverage (15.81) as indicated by the $95 \%$ confidence interval ( 0.55 , 0.66) on the difference in means which excludes zero.

Now turning our attention to respondents FSA adoption status, table (4) suggests that except for annual premium cost, all other quantitative predictors show significant mean differences between FSA subscribers and non-subscribers. Indeed, although individuals with FSA are on average significantly younger than those without FSA [43.7 years < 44.56 years; with 95\% C. I. $(-0.90$, $-0.27)]$, FSA subscribers have on average a significantly higher education level $[16.75>15.96$; with $95 \%$ C. I. ( $0.73,0.85$ )] and work significantly longer hours [41.95 hours $>41.23$ hours; with $95 \%$ C. I. $(0.41,1.01)]$. All of the described numerical results in this section are further confirmed by the eight graphical box plots representations in figure (1).

\subsection{Unadjusted v.s. Adjusted Uni-variate Models}

The single equation unadjusted probit (UP) and adjusted probit (AP) results are shown in table (5) for the two binary responses, optional coverage (SSP) and flexible spending accounts (FSA). In light of the results, we see that insurance premium cost has a positive and significant effect on the probability of adopting both SSP and FSA, although this effect is fairly negligible 
in the unadjusted FSA model. The results further suggest that not accounting for the effects of other relevant covariates biases the estimated effect of insurance premium cost on the two dependent variables, and even more so for FSA adoption. indeed the adjusted models suggest that insurance premium is not the only relevant determinant of SSP and FSA adoption in post-ACA USA, other factors such as health, socio-economic and demographic characteristics also affect these relationships. The relevance of these factors is further confirmed by the AIC and BIC measures produced by the UP and AP models. In fact, based on these model performance measures, we see that the AIC and BIC of the AP-SSP model are smaller than those of the UP-SSP model, and similarly for the AIC and BIC of the AP-FSA model compared to their counterparts in the UP-FSA model. Hence we can conclude that although the single equation adjusted probit specifications assume independence between SSP and FSA, they provide for a better model representation over the unadjusted models.

\subsection{Adjusted Uni-variate v.s. Fully-parametric Bivariate Models}

We now proceed to compare the results of the single equation adjusted probit models (AP-SSP, and AP-FSA) which implicitly assume independence between SSP and FSA, with those obtained by relaxing this independence assumption through the fully-parametric bivariate probit specification. This comparison of the two frameworks provide a formal test for the assumption of concurrent enrollment in SSP and FSA based on the significance of the correlation coefficient $\sigma_{12}$ between the two processes, as described in equation (14). The model is estimated using Heckman (1979) twostep approach with maximum likelihood, as implemented in the R library (Wojtys et al., 2016), which estimates the bi-variate model along with the correlation coefficient $\theta=\sigma_{12}$. The results at the bottom of table (6) suggest a positive and significant correlation coefficient $\theta=\sigma_{12}=0.147$ with $95 \%$ confidence interval $(0.127,0.165)$. This result indicates that the unobserved factors affecting individuals' decisions about optional coverage enrollment positively affect their decisions about FSA adoption, therefore the joint bivariate probit specification is more appropriate than the independent univariate binary probit models. This conclusion is further supported by the AIC measures produced by the models. Indeed the joint AIC values of the independent binary probit models $(43236.1+34121.8=77357.9)$ is greater than the AIC of the joint bivariate probit model (76723.8), hence relaxing the independence assumption through the latter specification is more appropriate for representing optional coverage and flexible spending accounts adoption as previously suggested in the theoretical discussion.

\subsection{Fully-parametric v.s. Semi-parametric Bi-variate Models}

The fully parametric bivariate probit and semi-parametric bivariate probit specifications both relax the implicit independence assumption in the binary probit equations. The latter further relaxes the linearity assumption made in the former model, and relies for identification on penalized maximum likelihood methods of estimation, which uses thin plate regression splines with basis 
dimensions 10 and penalties based on second order derivatives as described in Marra and Radice (2013). This estimation procedure deals simultaneously with the dependence between optional coverage and FSA using a conventional system of two equations, with non-linear covariate effects using spline approach, and also copes with non-normal bivariate distributions using copula functions (McGovern et al., 2015; Niankara, 2018a). The results of these two bivariate specifications are presented in table (7). Comparing the models' AICs and BICs measures at the bottom of table, we note that the semi-parametric specification with $\mathrm{AIC}=76409.85$, and $\mathrm{BIC}=77142.69$, performs better than the fully-parametric specification with $\mathrm{AIC}=76723.8$ and $\mathrm{BIC}=77151.48$. Therefore we choose the semi-parametric model as our preferred specification for describing the determinants of optional coverage (SSP) with Flexible spending accounts (FSA), and hence proceed to describe its results in the next section.

\subsection{Semi-parametric Bi-variate model results}

Focusing on the semi-parametric bivariate probit model, the convergence diagnostic checks for the trust region iteration algorithm (see (Wojtys et al. 2016) ) used to identify the parameters of the model, shows satisfactory convergence as the largest absolute gradient value $(1.528582 \mathrm{e}-10)$ is close to zero, and the observed information matrix is positive definite. The results are presented in table (7), and suggest that the unobserved factors affecting the likelihood of optional coverage positively correlate with those affecting the likelihood of FSA adoption. This is consistent with the results obtained under the fully-parametric specification, and shows a significant correlation coefficient $\theta=\sigma_{12}=0.141$ with a $95 \%$ confidence interval $(0.121,0.158)$.

\subsubsection{The Optional Coverage Equation: SPBP-SSP}

Semi-parametric bivariate probit estimation results for the optional coverage equation is shown in the second column of table (7). Focusing first on the effects of the nominal variables, it can be noted that factors such as physical health status, family medical expenditure, mental or emotional limitations, gender, race, marital status, region of residency, place of birth, citizenship, and Annual earnings are all significant factors in determining individuals choice of optional coverage.

In fact, starting with physical health status, we note that compared to respondents with excellent physical health status, individuals with very good, good, and fair health statuses are respectively $5.6 \%, 8.1 \%$, and $4.4 \%$ less likely to have optional coverage, although the effect for those with a fair physical health status is not significant. This result indicates that the more respondents report deteriorating physical health status, the less likely they are to have optional coverage. A potential explanation for this result is that optional coverage by granting access and usage to more health services including preventive health services, allows for better self reported health outcomes, than basic coverage. Table (7) Also shows that compared to respondents with less than $\$ 500$ in annual family medical expenditure, those with expenditure between $\$ 500$ and $\$ 2000$, and those with expenditure above $\$ 2000$ are respectively $8.9 \%$ and $12.6 \%$ less likely to have optional 
coverage. This finding suggests that the greater the annual medical expenditure, the less likely is an individual to have optional coverage. This result at first glimpse seems counter-intuitive, but might potentially be explained by the fact that individuals that lack optional coverage tend to incur greater out-of-pocket expenditure over the year when their realized health care needs are not covered by their basic insurance plan, while those with optional coverage are able to mitigate further out-of-pocket expenses, over and above that covered by their basic plan.

Furthermore, table (7) indicates that compared to respondents that report being limited, those not limited in anyway physical, mental or emotional are $14.2 \%$ more likely to have optional coverage. This result seems to further confirm the fact that respondents with optional coverage are better able to access and use more preventive health services leading to better physical, mental and emotional health outcome overall, compared to their counterparts without optional coverage. Moreover, the results in table (7) show that compared to male respondents, females are $12.5 \%$ less likely to have optional coverage. This result seems to indicate the existence of a gender based inequality in access to extended coverage in post-ACA USA. Although ACA mandates have made gender based premium discrimination illegal, this result seems to suggest that females are yet to take advantage of this health care statute and get as much health coverage at reasonable costs as their male counterparts.

In addition, the results in table (7) indicate that compared to Caucasian (white) respondents, Asians are $10.6 \%$ less likely to have optional coverage, while blacks and other races are respectively $7.2 \%$ and $14.7 \%$ more likely to have optional coverage. With respect to marital status, we note that compared to currently married respondents, those previously married, and those that never married are respectively $7.3 \%$ and $9.8 \%$ more likely to have optional coverage. With respect to the regional variables, we note that compared to respondents from the south, those living in the Northwest, Midwest, and West are respectively $16.3 \%, 12.7 \%$ and $4.6 \%$ less likely to have optional coverage in post- ACA USA.

With regards to respondent place of birth, the results show that compared to respondents born inside the US, those born outside of the United States are 24.3\% more likely to have an optional coverage. Similarly, compared to US citizens, non citizens are $13.2 \%$ more likely to have optional coverage. Looking at respondent earnings, the results suggest that compared to those with earnings below $\$ 35000$, those with annual earnings between $\$ 35000$ and $\$ 65000$ and those with earnings above $\$ 65000$ are respectively $14.3 \%$ and $31.6 \%$ less likely to have optional coverage. These results suggest that as earnings increase respondents' likelihood of adopting an optional coverage decreases.

Now turning to the smoothed terms for the quantitative variables in the optional coverage equation, it can be noted that insurance premium cost, age, education level, and weekly hours worked are all statistically significant predictors of individual's likelihood of extending coverage beyond the basic coverage. Indeed the p-values $(<0.05)$ and estimated empirical density function (edf) in the second column of table (7), along with the respective smooth functions estimates 
and $95 \%$ confidence bands in the upper panel of figure(2) validate their statistical significance in explaining the phenomenon of optional coverage adoption among the privately insured in post-ACA USA.

The first graph in the upper panel of figure (2) suggests that at annual premium costs below $\$ 5000$, insurance premium impacts negatively the likelihood of optional coverage; above the annual premium cost of $\$ 5000$, an increase in premium cost raises the likelihood of optional coverage, and does so at an increasing rate, therefore suggesting that premium cost have a non-linear effect on the probability of optional coverage enrollment. The second graph in the upper panel of figure (2) which describes the effect of age, does indeed show that below age 52, an increase in respondents age has a negative impact on this likelihood of optional coverage enrollment, however past this threshold, increasing age significantly increases this likelihood suggesting therefore that age does also have a non-linear influence on optional coverage choice. The third graph in the upper panel of figure (2) describes the effect of education level on the likelihood of optional coverage enrollment, and suggest that at levels of education below 15, increasing education level have a positive but decreasing effect on the likelihood of optional coverage enrollment; however pass this threshold, the effect of education is negative and significant. The fourth and last graph in the upper panel of figure (2) depicts the effect of weekly hours spent working on the likelihood of optional coverage enrollment. We note that below 40 hours, the effect of increasing hours of work on optional coverage enrollment is positive but decreasing, between 40 to 50 hours this effect in negative and convex, above 50 hours the effect is positive and increasing. Overall, the smooth function estimates along with the $95 \%$ confidence bands support and confirm the significance of the semi-parametric framework over its fully-parametric counterparts that assume the effects of the included numerical co-variates to be linear, and thereby failing to capture the true non-linearities in these effects.

\subsubsection{The Flexible Spending Accounts Equation: SPBP-FSA}

The semi-parametric bivariate probit estimation results for the FSA equation is shown in the last column of table (7). Focusing first on the effects of the nominal variables as before, it can be noted that except for physical health status, mental or emotional limitations, factors such as family medical expenditure, gender, race, marital status, region of residency, place of birth, citizenship, and annual earnings are all significant determinants of individuals' choice of Flexible Spending Accounts (FSA) in post-ACA USA.

In fact, starting with medical health expenditure, table (7) shows that compared to respondents with less than $\$ 500$ in annual family medical expenditure, those with expenditure between $\$ 500$ and $\$ 2000$ and those with expenditure above $\$ 2000$ are respectively $19.3 \%$ and $41.7 \%$ less likely to have a FSA. This finding suggests that the greater the annual family medical expenditure, the less likely is an individual to have a FSA. This result at first glimpse seems counter-intuitive, but might be explained by the fact that individuals that lack a FSA tend to incur greater out-of-pocket expenditure over the year when their realized health care needs are not covered by their current 
insurance plan, while those with FSA, are able to mitigate annual out-of-pocket expenses using their tax free FSA funds.

Furthermore, table (7) indicates that compared to male respondents, females are $7.0 \%$ less likely to have a FSA. This result seems to indicate the existence of gender based inequality in FSA account adoption in post-ACA USA. In addition, table (7) indicates that compared to Caucasian (white) respondents, blacks and Asians are respectively 6.3\% and 9.1\% less likely to have a FSA. With respect to marital status, we note that compared to currently married respondents, those previously married, and those that never married are respectively $14.6 \%$ and $9.3 \%$ more likely to have a FSA. The regional distribution of the respondents, show that compared to respondents from the south, those living in the Northwest, Midwest, and West are respectively 7.0\%, 14.5\% and $4.7 \%$ less likely to adopt a FSA in post- ACA USA.

With regards to the place of birth, table (7) shows that compared to respondents born inside the US, those born outside of the United States are 19.1\% more likely to adopt FSAs. Similarly, compared to US citizens, non citizens are $17.5 \%$ more likely to have FSAs. Looking at respondent earnings, the results suggest that compared to those with earnings below $\$ 35000$, those with annual earnings between $\$ 35000$ and $\$ 65000$ and those with earnings above $\$ 65000$ are respectively $21.7 \%$ and $40.2 \%$ less likely to have FSAs. These results suggest that as earnings increase respondents' likelihood of adopting a flexible spending accounts decrease.

Now turning to the smoothed terms for the quantitative variables in the FSA equation, it can be noted that insurance premium cost, age, education level, and weekly hours worked are all statistically significant predictors of individual's likelihood of having a flexible spending account. Indeed the p-values $(<0.05)$ and estimated empirical density function (edf) in the last column of table (7), along with the respective smooth functions estimates and $95 \%$ confidence bands in the lower panel of figure(2) validate their statistical significance in explaining the phenomenon of FSA adoption among the privately insured in post-ACA USA.

The first graph in the upper panel of figure (2) suggests that at annual premium cost below $\$ 5000$, insurance premium impacts negatively the likelihood of FSA adoption; above the annual premium cost of $\$ 5000$, an increase in premium cost raises the likelihood of FSA adoption, and does so at an increasing rate, therefore suggesting that premium cost have indeed a non-linear effect on the probability of FSA adoption. The second graph in the lower panel of figure (2) which describes the effect of age, does indeed show that below age 50, an increase in age has a negative impact on the likelihood of FSA adoption, between 50 and 60, age has no effect on FSA adoption, however above 60 increased age significantly raises the likelihood of FSA adoption, hence suggesting that age does also have a non-linear influence. The third graph in the lower panel of figure (2) describes the effect of education level on the likelihood of FSA adoption, and suggests that at levels of education below 15, education level has a positive but quadratic effect on the likelihood of FSA adoption; however past this threshold, the effect of education is negative and significant. The fourth and last graph in the lower panel of figure (2) depicts the effect of weekly 
hours spent working on the likelihood of FSA adoption. We note that below 20 hours, the effect of increasing hours of work on FSA adoption is positive but decreasing, between 20 to 45 hours this effect in negative and convex, above 45 hours the effect is positive and increasing. Overall, the smooth function estimates along with the $95 \%$ confidence bands support and confirm the significance of the semi-parametric framework over its fully-parametric counterparts that assume linearity of the numerical covariates such as age, and hours worked, and therefore fail to capture and describe the non-linearities in these effects.

\section{Conclusion}

This paper has concerned itself with analyzing the determinants of optional coverage (SSP) and flexible spending accounts (FSA) enrollment by the privately insured in post-ACA USA, using the pooled cross-sectional survey of the 2015-2016 National Health Interview Surveys. The study was motivated by the theoretical model of health insurance choice with Flexible Spending Accounts (FSAs) presented in Cardon (2012). To achieve our study goal, the empirical strategy adopted consisted of sequentially contrasting various specifications for SSP and FSA enrollment. Based on model performance measures, the semi-parametric bivariate probit specification was identified as the best framework for describing the factors affecting optional coverage and FSA enrollment.

Overall the results identified several factors as significant determinants of this joint probability, including insurance premium cost, family annual medical expenditure, gender, race, marital status, age, region of residence, place of birth, citizenship, education, annual earnings, and hours spent working. By identifying these factors the current study places itself as one of the few to investigate the determinants of flexible spending accounts adoption in the period post-ACA USA, but most importantly one of the few to report on the welfare implications of the changes brought by the ACA tax exclusion limitations on FSA contributions to a maximum of $\$ 2600$ (2017 \$s). This limitation when first imposed was estimated to increase federal payroll and income tax receipts by $\$ 13$ billion between 2013 and 2019, however its welfare implications have remained fairly undocumented.

Contrasting our results with the findings in Cardon et al. (2012) who explored patterns of FSA usage by income and health status, and concluded that household likely to be affected by ACA limitations are those experiencing chronic or deteriorating health conditions. They further justified this by the fact that chronic illness is associated with relatively high and persistent medical expenses such that FSA tax exclusion limitations would mean greater out-of-pocket expenditure even with FSA. Here our results showed that controlling for annual medical expenditure and other relevant socio-economic and demographic factors, physical, mental, or emotional health status do not seem to have significant effects on FSA adoption in post-ACA USA. However, the negative and increasing effect of medical expenditure on FSA adoption corroborates with their conclusions as seemingly indicating that because of the legislated limitations on FSA tax exclusions, individuals with higher medical expenses have a lesser incentive to enroll in optional coverage and flexible 
spending accounts in post-ACA USA. Further corroborating with their conclusions are the negative effects of increased annual earnings observed here on both optional coverage and FSA enrollment potentially due in part to the adverse welfare effect of the exclusion limitations.

Additionally, our result of the effect of race, are also in line with Hamilton and Marton (2008) which also examined the determinants of FSA participation, and found that even after controlling for other demographic characteristics, non-whites are less likely to participate in the FSA program than their white counterparts. Here we found that blacks and Asians are respectively $6.3 \%$ and $9.1 \%$ less likely to enroll in flexible spending accounts compared to whites in post-ACA USA.

Finally, beyond being the first to report on the non-linearity of the effects of key determinants of optimal coverage and FSA enrollment, this study provides one of the most up to date treatments of FSA demand at the extensive margin using a nationally representative sample of the privately insured population in the USA. Understanding these factors is key to providing tailored solutions for reducing health risks, and therefore is of interest to all US health care market stakeholders.

\section{References}

Cameron, A Colin and Pravin K Trivedi (2005), Microeconometrics: methods and applications, Cambridge university press.

Cardon, James H (2012), 'Health insurance choice with flexible spending accounts', The Geneva Risk and Insurance Review 37(2), 208-222.

Cardon, James H, Jeffrey T Denning and Mark H Showalter (2013), 'Flexible spending accounts and the use-it-or-lose-it provision', Applied Economics 45(35), 4928-4939.

Cardon, James H, Joel Moore and Mark H Showalter (2012), The distributional effects of health reform limits on flexible spending accounts, in 'Forum for Health Economics \& Policy', Vol. 15, De Gruyter.

Cardon, James H and Mark H Showalter (2001), 'An examination of flexible spending accounts', Journal of Health Economics 20(6), 935-954.

Cardon, James H and Mark H Showalter (2003), 'Flexible spending accounts as insurance', Journal of Risk and Insurance 70(1), 43-51.

Dranove, David, Kathryn E Spier and Laurence Baker (2000), 'Competition'among employers offering health insurance', Journal of Health Economics 19(1), 121-140.

Even, William E and David A Macpherson (2016), 'The affordable care act and the growth of involuntary part-time employment'. 
Feldman, Roger and Jennifer Schultz (2001), 'Who uses flexible spending accounts: effects of employee characteristics and employer strategies', Medical care 39(7), 661-669.

Gruber, Jonathan and Robin McKnight (2003), 'Why did employee health insurance contributions rise?', Journal of Health Economics 22(6), 1085-1104.

Hamilton, Barton H and James Marton (2008), 'Employee choice of flexible spending account participation and health plan', Health Economics 17(7), 793-813.

Harrington, Scott E (2010), 'Us health-care reform: The patient protection and affordable care act', Journal of Risk and Insurance 77(3), 703-708.

Heckman, James J. (1979), 'Sample selection bias as specification error', Econometrica 47(1), 153161.

Jack, William, Arik Levinson and Sjamsu Rahardja (2006), 'Employee cost-sharing and the welfare effects of flexible spending accounts', Journal of Public Economics 90(12), 2285-2301.

Manski, Charles F. and Daniel L. McFadden (1981), Structural Analysis of Discrete Data and Econometric Applications, The MIT Press, Cambridge.

Marra, Giampiero and Rosalba Radice (2013), 'Estimation of a regression spline sample selection model', Computational Statistics \& Data Analysis 61, 158-173.

Marshak, Jacob (1959), 'Binary choice constraints on random utility indications', Stannford Symposium on Mathematical Methods in the Social Sciences. available at http://www.cowles.econ.yale.edu/p/cp/p01b/p0155.pdf.

McGovern, Mark E, Till Bärnighausen, Giampiero Marra and Rosalba Radice (2015), 'On the assumption of bivariate normality in selection models: a copula approach applied to estimating hiv prevalence', Epidemiology (Cambridge, Mass.) 26(2), 229.

National Center for Health Statistics and others (2015), '2015 national health interview survey (nhis) public use data release: Nhis survey description', Division of Health Interview Statistics, National Center for Health Statistics, Centers for Disease Control and Prevention. Available from the NHIS Web site (www. cdc. gov/nchs/nhis. htm).

National Center for Health Statistics and others (2016), '2016 national health interview survey (nhis) public use data release: Nhis survey description', Division of Health Interview Statistics, National Center for Health Statistics, Centers for Disease Control and Prevention. Available from the NHIS Web site (www. cdc. gov/nchs/nhis. htm). 
Niankara, Ibrahim (2018a), 'Gender inequality in literacy status, and its effects on households economic well-being in burkina faso: A semiparametric bi-variate sample selection modeling approach', International Journal of Economics Business Research forthcoming.

URL: http://www.inderscience.com/info/ingeneral/forthcoming.php?jcode=ijebr

Niankara, Ibrahim (2018b), 'Health and environmental consciousness effects of wealth in low income countries: Evidence from households' energy, water, and sanitation services consumption in burkina faso', Preprints, 2018020121 (doi: 10.20944/preprints201802.0121.v1). pp. 1-26.

Niankara, Ibrahim (2018c), 'Organizational management culture and employers' health insurance offering strategies in the us: An ubuntu based random utility modeling approach', Global Business and Economics Review 20(4), 503-520.

Niankara, Ibrahim (2018d), 'The relative influence of inter-generational co-residence on healthcare market and labor market outcomes in post affordable-care-act usa', Global Business and Economics Review forthcoming.

URL: http://www.inderscience.com/info/ingeneral/forthcoming.php?jcode=GBER

Pauly, Mark V (1986), 'Taxation, health insurance, and market failure in the medical economy', Journal of Economic Literature 24(2), 629-675.

R Core Team (2015), R: A Language and Environment for Statistical Computing, R Foundation for Statistical Computing, Vienna, Austria.

URL: https://www.R-project.org/

Ramsay, Colin M and Victor I Oguledo (2011), 'Optimum allocations to health care flexible spending accounts', North American Actuarial Journal 15(3), 448-467.

Train, Kenneth E. (2009), Discrete Choice Methods with Simulation, Cambridge University Press, New York.

Wojtys, Malgorzata, Giampiero Marra and Rosalba Radice (2016), 'Copula regression spline sample selection models: the r package semiparsamplesel', Journal of Statistical Software 71(6). 

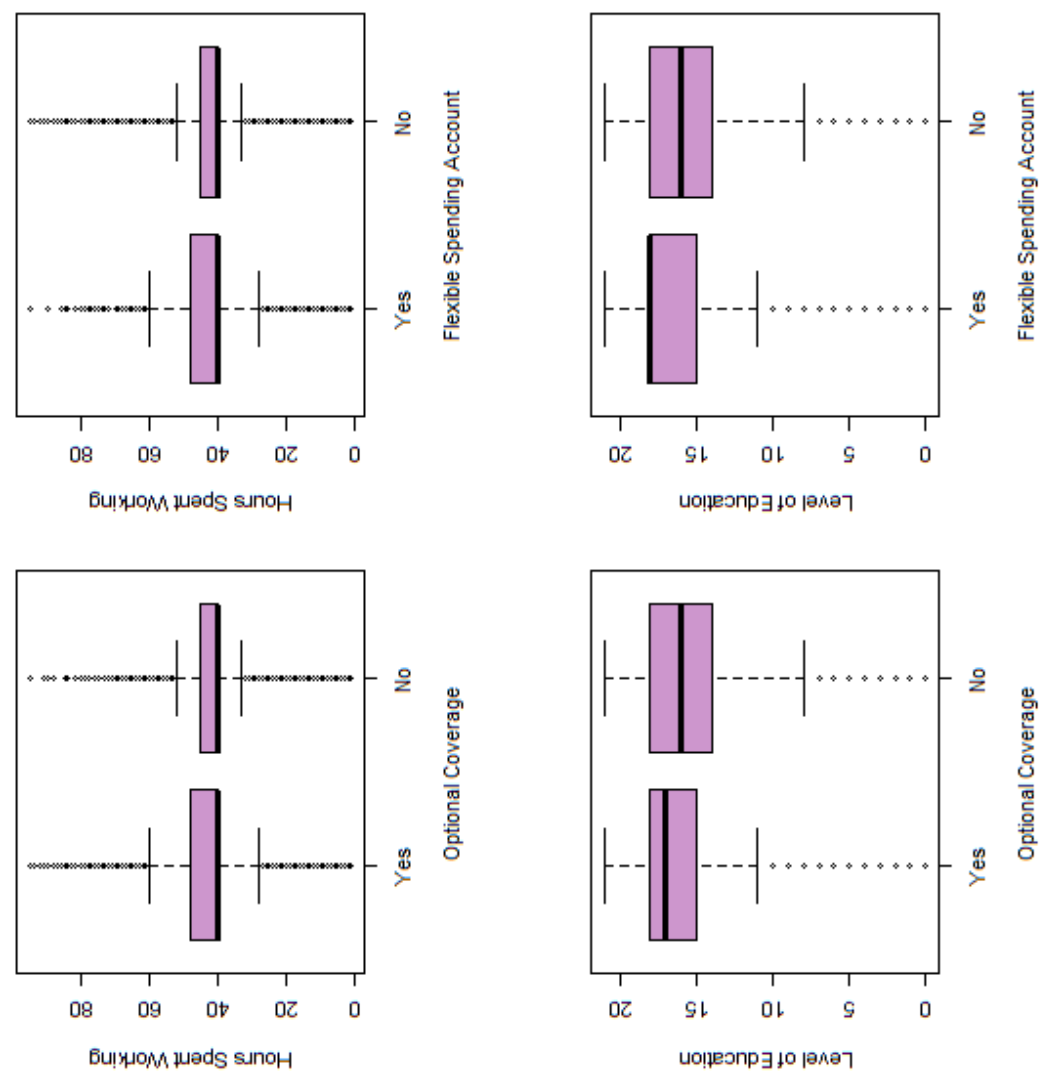

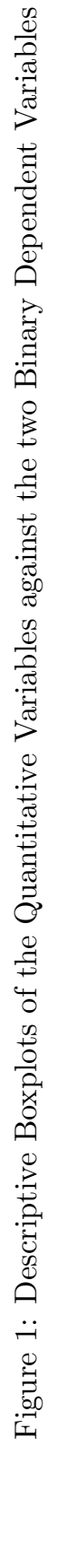




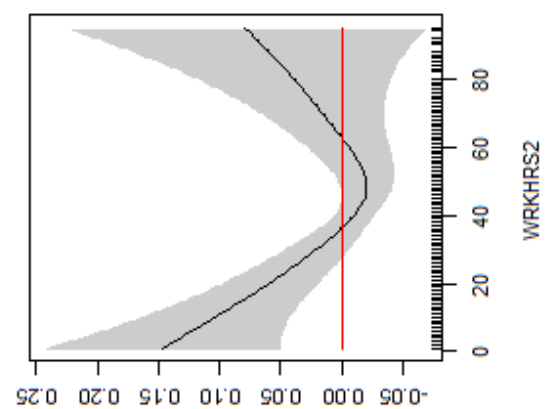

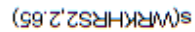
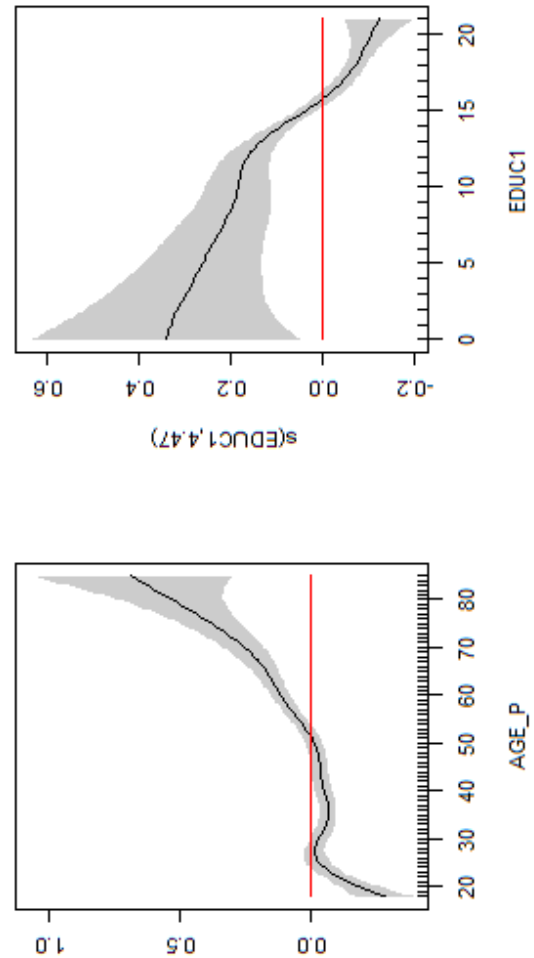

( IZ L'd $\left.{ }^{\top} \exists O V\right) \mathrm{s}$

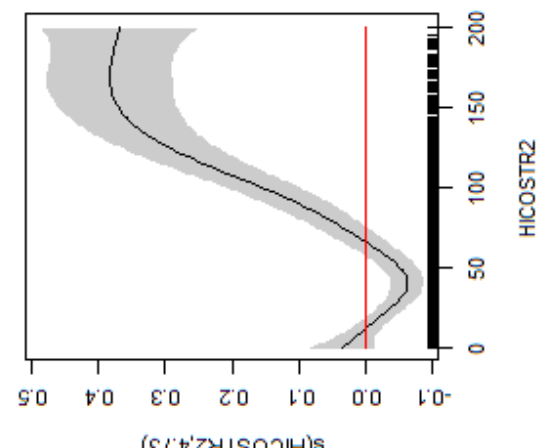

(EL't'Zy

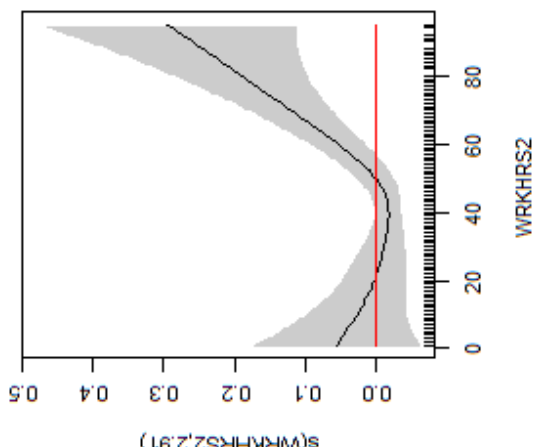

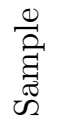

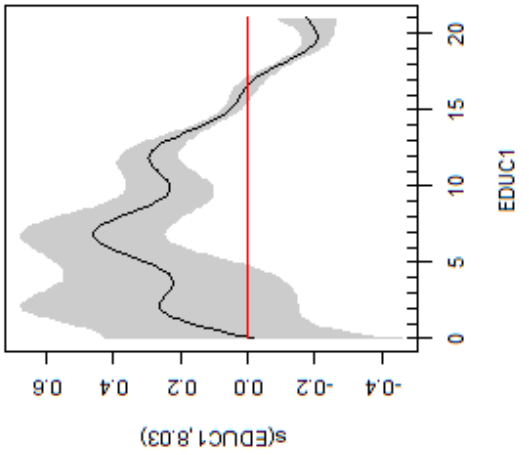

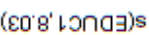
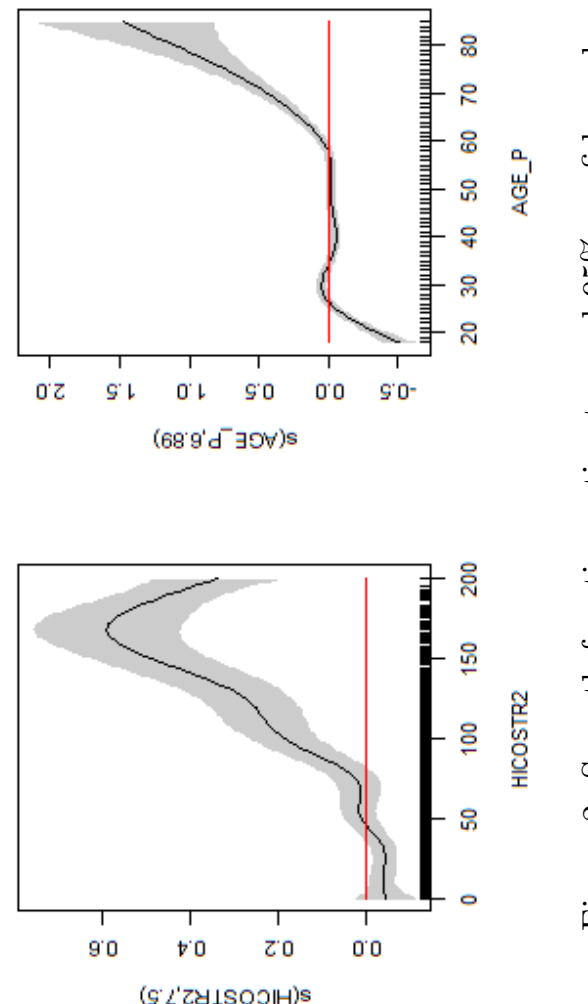

(c'L'ZYISOJH)s 